\title{
Les enjeux du signalement d'un mineur
}

\section{Madeleine Mirabaud}

Médecin adjointe, Groupe de Protection de I'enfant, Hôpitaux Universitaires de Genève
Les professionnels de la santé peuvent être confrontés à des situations complexes et délicates où ils constatent que le développement d'un enfant est sérieusement menacé. Il peut s'agir de mise en danger de l'enfant sur le plan physique, psychologique, sexuel, mais aussi de retard de développement de l'enfant, de négligence matérielle grave ou de problèmes importants de collaboration avec les parents.

Depuis quelques années, les dispositions légales concernant les possibilités et obligations de signalements de mineurs en danger sont de plus en plus nombreuses, et suscitent des discussions entre professionnels, notamment en ce qui concerne les conséquences positives et négatives des signalements.

\section{Signalement}

Le signalement à une autorité de protection ou à un service de protection des mineurs est une décision médicale parfois difficile, car elle est au carrefour d'aspects multiples: médicaux, légaux, sociaux, psychologiques, éthiques, ...

Une réflexion approfondie autour de certains de ces enjeux semble nécessaire pour éviter de réduire le signalement à un conflit opposant secret médical et protection de l'enfant. Il est également important de distinguer ces aspects en fonction du rôle du médecin qui signale. Dans un même cas de figure, les enjeux d'un signalement seront différents pour un pédiatre installé en cabinet, un médecin scolaire, un médecin spécialiste ou un médecin hospitalier, car certains aspects peuvent paraître primordiaux pour les uns et moins importants pour d'autres.

\section{Du point de vue médical}

Le médecin confronté à une atteinte avérée ou à un risque sérieux d'atteinte au bien-être d'un enfant doit toujours commencer par relever précisément les faits et circonstances observés et relatés qui sont inquiétants. Même si dans certains cas l'atteinte à l'intégrité psychique ou physique de l'enfant est grave et nécessite des mesures urgentes, dans la majorité des cas le médecin doit prendre du temps pour mieux comprendre la situation. Après avoir procédé à une analyse complète de la situation, le médecin doit activement chercher à collaborer avec le patient et sa famille et proposer des mesures permettant d'améliorer la situation dans l'intérêt de l'enfant. Si une telle collaboration s'avère impossible, ou si la situation apparaît d'emblée très grave, le médecin doit envisager le signalement, en essayant de déterminer quelles interventions externes pourront permettre une amélioration. Il convient donc d'expliciter dans le signalement les raisons ayant mené à cette démarche et d'établir une demande précise de mesures tout en veillant au principe de proportionnalité. Le signalement doit s'inscrire dans la mesure du possible dans une démarche transparente par rapport au patient et à ses parents.

Il faut aussi rappeler qu'avant de signaler, le médecin doit veiller à s'assurer qu'il n'y aura pas de mise en danger imminente de l'enfant suite au signalement.

\section{Du point de vue légal}

Les faits qui vont conduire le médecin à procéder à un signalement ont été portés à la connaissance du médecin dans le contexte de la relation médecin-malade. Cette relation est protégée par la notion de secret médical, car une relation de confiance suffisante doit exister entre le patient et son médecin pour que l'exercice de la médecine puisse se faire de façon satisfaisante. Ce secret professionnel est inscrit dans le Code pénal suisse (art. 321). Les professionnels de la santé y sont tenus, qu'ils soient en pratique privée ou institutionnelle, ainsi que leurs auxiliaires.

Le médecin est aussi tenu au secret de fonction au sens de l'art. 320 du Code pénal suisse quand il travaille dans un établissement public, et peut en être délié avec le consentement de l'autorité à laquelle il est subordonné. Quand la levée du secret professionnel ne peut pas être obtenue et que la commission d'une infraction pénale à l'encontre d'un mineur est suspectée, la législation fédérale prévoit la possibilité de signalement à l'autorité de protection en vertu de l'art. 364 du Code pénal suisse. Il n'y a alors pas besoin de levée de secret par le patient (ou par son représentant légal en cas d'incapacité de discernement).

Plus récemment, l'art. 443 du Code civil suisse, entré en vigueur le $1^{\text {er }}$ janvier 2013, prévoit à l'alinéa 1 que «toute personne a le droit d'aviser l'autorité de protection qu'une personne semble avoir besoin d'aide. Les dispositions sur le secret professionnel sont réservées.» La levée 
du secret médical est donc nécessaire et peut se faire par le patient capable de discernement ou par le représentant légal du patient incapable de discernement. En cas d'absence de levée du secret, une demande de levée peut être faite à l'autorité compétente (par exemple à Genève la commission de levée du secret professionnel).

A l'alinéa 2 de l'art. 443 du Code civil suisse, il est prévu que toute personne dans l'exercice de sa fonction officielle a l'obligation d'informer l'autorité, et qu'il peut y avoir d'autres obligations cantonales d'aviser l'autorité. Les législations cantonales ont dû s'adapter à cette nouvelle législation fédérale et prévoir dans quels cas il est obligatoire de renseigner d'autres services et lesquels. Chaque canton a donc une loi d'application qui prévoit quand et comment informer l'autorité. Par exemple à Genève, la levée du secret reste nécessaire, même si la loi prévoir l'obligation de signalement au Service de protection des mineurs (art. 34 de la loi d'application du Code civil).

\section{Du point de vue éthique}

La notion de confidentialité est au cœur de la relation médecin-malade et a pour but de préserver la relation de confiance entre le patient et son médecin. En abordant avec le patient mineur (ou avec le représentant légal de ce dernier en cas d'incapacité de discernement) les atteintes à la santé ou au développement, le médecin veille au respect de l'autonomie du patient, en l'informant et en l'incluant dans le processus de prise en charge.

Toutefois, si le patient (ou son représentant légal) ne partage pas le point de vue du médecin ou ne comprend pas l'inquiétude énoncée par le médecin, le principe d'autonomie va rapidement entrer en conflit avec le principe de bienfaisance. En effet, le médecin a pour objectif le bien de l'enfant. En voulant préserver l'intérêt de l'enfant et mettre en avant le principe de bienfaisance, un conflit de valeurs entre autonomie et bienfaisance va apparaître. Le médecin, quel que soit son rôle, va prendre des décisions dans l'intérêt de l'enfant qui n'a pas la capacité de discernement suffisante pour prendre position lui-même. C'est dans ce contexte que le signalement s'inscrit, d'autant plus que les parents sont souvent eux-mêmes impliqués dans les difficultés présentées par l'enfant, et qu'il semble difficile de se baser sur leur point de vue quand il va clairement à l'encontre de l'intérêt de l'enfant.

Par ailleurs, le principe de bienfaisance doit être contrebalancé par le principe de non-malfaisance car le médecin doit éviter de prendre des décisions qui pourraient avoir un effet négatif pour le mineur.

Correspondance:

Madeleine Mirabaud

Hôpitaux Universitaires

de Genève

6, rue Willy Donzé

CH-1211 Genève 14

madeleine.mirabaud[at]

hcuge.ch de curatelle. Même si le médecin travaille régulièrement avec d'autres professionnels, la prise en charge par un service de protection des mineurs peut apporter une aide précieuse dans un grand nombre de situations. Le réseau devient alors un élément important pour la prise en charge d'un enfant et sa sécurité.

Le médecin ne doit pas décider d'un signalement dans le but de transférer sa responsabilité à un autre professionnel par rapport à une situation à risque, voire se décharger de sa responsabilité. Il doit signaler pour introduire la collaboration avec d'autres spécialistes de la protection de l'enfant.

\section{Du point de vue psychologique}

Le signalement peut avoir des conséquences psychologiques non négligeables sur la relation médecin-malade, mais aussi sur tout le système familial. En effet, par le recours à un tiers, la relation de confiance peut être mise à mal. Il faut donc prendre du temps pour préparer le patient et sa famille à la nécessité d'un signalement, obtenir si possible leur accord, et partager le contenu exact de la transmission d'informations.

\section{Evolution}

La législation actuelle tend vers l'obligation de signaler. L'art. 443 du Code civil suisse, entré en vigueur en 2013, a introduit l'obligation de signaler. Même si cette obligation est énoncée dans le respect du secret professionnel, elle apporte un changement fondamental dans la perception globale des situations dans lesquelles des mineurs sont en danger. Jusqu'à ce jour, le droit fédéral avec l'art. 364 CP émettait uniquement la possibilité de signalement. Le droit fédéral est actuellement interprété par certains médecins dans l'idée que l'article 443 du Code civil, étant plus récent que l'art. $364 \mathrm{CP}$, prime sur ce dernier qui donnait uniquement la possibilité de signaler. Un projet de modification du Code civil est en cours et, dans le cadre de la procédure de consultation, de nombreuses réserves sont émises par rapport aux difficultés liées à l'introduction d'une obligation de signalement.

\section{Conclusion}

Les médecins en charge de patients mineurs doivent identifier les situations à risque et prendre toutes les mesures nécessaires dans l'intérêt des mineurs. Ils sont tenus dans le cadre de leur profession à certaines obligations légales, et doivent toujours veiller à une pesée d'intérêts afin d'agir dans l'intérêt de l'enfant et de sa famille.

La relation de confiance qui existe entre un patient et son médecin est un aspect important de l'exercice de la médecine et doit rester au premier plan de la prise en charge des mineurs. 